\title{
Avaliação ultrassonográfica do bulbo ocular em cães submetidos à facoemulsificação com ou sem implante de lente intraocular
}

\author{
[Ocular bulb ultrasonographic evaluation in dogs submitted to facectomy by facoemulsification \\ with or without intraocular lens implants]
}

\author{
P.T. Pavan, J.J.T. Ranzani, M.F. Almeida, M.J. Mamprim, C.V.S. Brandão
}

Faculdade de Medicina Veterinária e Zootecnia - Unesp - Botucatu, SP

\begin{abstract}
RESUMO
Este trabalho objetivou descrever e comparar as alterações ultrassonográficas encontradas em bulbos oculares de cães submetidos à facoemulsificação, com ou sem implante de lente intraocular (LIO), no intuito de auxiliar no diagnóstico de alterações decorrentes da cirurgia e do implante da LIO, bem como sua correspondência com alterações clínicas. Dezenove cães portadores de catarata (21 olhos) foram submetidos ao procedimento de facoemulsificação e avaliados no pós-operatório tardio, aos cinco anos de evolução. Constituíram-se três grupos experimentais: afácicos (CA), formados por 11 cães; pseudofácicos (PP), com implante de duas LIOs em piggyback ( $\mathrm{n}=5$ ); e pseudofácicos (PL), com implante de uma LIO veterinária $(\mathrm{n}=5)$. Após exame oftálmico, todos foram submetidos ao exame ultrassonográfico, sob anestesia tópica, com transdutor linear multifrequencial de $10 \mathrm{MHz}$; adicionalmente foram avaliadas medidas biométricas dos bulbos do olho. As principais alterações observadas foram: luxação da LIO, descolamento de retina, hialose asteroide e degeneração vítrea. O exame ultrassonográfico mostrou-se como excelente ferramenta diagnóstica e possibilitou a confirmação e a classificação dessas alterações. $\mathrm{Na}$ biometria ocular, pelas medidas entre o corpo ciliar e a câmara vítrea, o implante em piggyback resultou em redução destas em relação aos demais procedimentos instituídos, não havendo diferença ente o comprimento axial e a câmara anterior.
\end{abstract}

Palavras-chave: cão, biometria ocular, olho, LIO

\begin{abstract}
This study aimed to describe and compare the ultrasonographic alterations in dogs' eyes submitted to facectomy with or without intraocular lens implant (IOL), to assist in the diagnosis of possible alterations related to the surgical procedure and IOL implantation. Nineteen dogs with cataract (21 eyes) were submitted to phacoemulsification and late postoperative evaluation (at five years). The animals were initially submitted to complete ophthalmological exams which preceded the sonogram. Dogs were divided in three groups: $(C A)$ aphakic dogs $(n=11) ;(P P)$ pseudophakic dogs with implantation of two IOLs in piggyback $(n=5)$ and (PL) pseudophakic dogs with implantation of a veterinary IOL $(n=5)$. The ultrasound was carried under the administration of a topic local anesthetic, with a multi frequency linear transducer of $10 \mathrm{MHz}$. Biometric eye measurements were also performed. The clinical alterations observed were IOL dislocation, retinal detachment, asteroid hyalosis, and vitreous degeneration. Ultrasound examination was an excellent diagnostic tool, as it was possible to confirm and classify these changes. The piggyback implant reduced the measurements between the ciliary body and the vitreous chamber obtained from the ocular biometry when compared to other usual procedures, with no difference between the axial length and the anterior chamber.
\end{abstract}

Keywords: dog, ocular biometry, eye, IOL

Recebido em 6 de abril de 2012

Aceito em 7 de maio de 2013

E-mail: quim@fmvz.unesp.br 


\section{INTRODUÇÃO}

A catarata é definida como uma opacificação da cápsula ou fibras da lente, decorrente de alterações da arquitetura lamelar dessas estruturas. A afecção ocupa lugar de destaque na oftalmologia veterinária, sendo uma das principais causas relacionadas à perda de visão no cão (Glover e Constantinescu, 1997; Slatter, 2005; Gelatt, 2007).

As técnicas cirúrgicas utilizadas para remoção da catarata e LIOs têm evoluído rapidamente, sempre no intuito de aumentar as taxas de sucesso do procedimento, com diminuição das complicações. Atualmente, é consenso mundial a abordagem cirúrgica como único tratamento da catarata, sendo que a técnica de escolha para remoção da catarata é a facoemulsificação, a qual consiste na fragmentação da lente, com posterior aspiração do material emulsificado, por meio de uma pequena incisão de $3 \mathrm{~mm}$ ou inferior (Davidson et al., 1991; Nasisse et al., 1991; Bistner, 1992; Williams et al., 1996; Glover e Constantinescu, 1997; Gelatt, 2007).

As principais complicações advindas da cirurgia para extração da catarata incluem as uveítes anteriores, hifema, edema de córnea, opacidade de cápsula, descolamento de retina e glaucoma, o que resulta em opacificação dos meios, impossibilitando o exame dos segmentos anterior e posterior (Dziezyc, 1990; Davidson et al., 1991; Whitley et al., 1993; Williams et al., 1996; Gelatt, 2007).

É fundamental a identificação de quaisquer alterações oculares que contraindicam a facectomia ou alteram o prognóstico visual dos pacientes, tais como a formação de coágulos e infiltrado celular no vítreo, a degeneração vítrea e o deslocamento da retina parcial ou total, entre outros. Sendo assim, o exame ultrassonográfico é imprescindível no diagnóstico dessas diferentes alterações, especialmente as do segmento posterior (Squarzoni, 2007).

O exame ultrassonográfico (ecografia) tem sido utilizado para diagnóstico das doenças oftálmicas em medicina desde 1956; já em medicina veterinária, o seu uso foi somente descrito em 1968 (Nyland e Mattoon, 2005; Kealy et al., 2012). O bulbo ocular é uma ótima estrutura para ser analisada pelo método, por ser, em grande parte, constituído de líquidos e superfícies internas refletivas; além de promover pouco estresse ao animal, também é seguro e não invasivo (Gonzalez et al., 2001; Carvalho, 2004; Kealy et al., 2012).

Apontado como exame de pré-triagem indispensável na detecção da catarata, por fornecer informações relevantes da lente, notadamente quanto ao seu aspecto e dimensões (Martins et al., 2010), e por verificar a estrutura acometida e a origem do surgimento da doença (Pontes e Corrêa, 2011), o ultrassom é excelente para avaliar estruturas orbitais e conteúdo intraocular obscurecido da visualização devido à opacidade da lente, córnea ou câmara anterior ou para avaliar possíveis causas de exoftalmia (Williams e Wilkie, 1996; Laus et al., 2003; Paiva, 2011). Consiste basicamente na utilização de uma probe com cristais piezoelétricos, sendo os ecos de retorno formados, filtrados, amplificados e exibidos na tela; permitem, pois, ao médico veterinário análise de porções individualizadas ("fatias") (Nyland e Mattoon, 2005).

A biometria ocular, em modo $\mathrm{B}$, promove a mensuração do comprimento axial do bulbo ocular, entre outras medidas; tem aplicação direta no cálculo do poder dióptrico da lente intraocular (LIO) em pacientes submetidos à facectomia, do tamanho do implante na substituição protética após enucleação, bem como possibilita a avaliação e a comparação em diversas afecções oftalmológicas (González, 2001; Dietrich, 2007; Peixoto et al., 2008; Paiva, 2011).

Diante dos aspectos citados anteriormente, a realização do presente experimento foi estimulada pela necessidade de avaliarem-se as alterações clínicas e as complicações intraoculares em cães acometidos por catarata, submetidos à facectomia por facoemulsificação com ou sem implante de lente intraocular, utilizando-se do exame ultrassonográfico para acompanhamento da evolução no pós-operatório tardio.

\section{MATERIAL E MÉTODOS}

Os procedimentos experimentais desenvolvidos foram submetidos à Câmara de Ética em Experimentação Animal da Faculdade de 
Medicina Veterinária e Zootecnia - Unesp Campus de Botucatu (Processo no 177/2008 CEEA).

Foram analisados 19 cães, machos e fêmeas, não diabéticos, de raças e idades variadas, afácicos ou pseudofácicos, totalizando 21 bulbos oculares. Integraram os grupos de estudo animais atendidos e submetidos ao procedimento de facoemulsificação em córnea clara, por um único cirurgião. Todos os animais foram avaliados previamente à cirurgia de facoemulsificação por meio de exame clínico oftalmológico e ultrassonográfico, como rotina. Nestes exames foi confirmada a catarata uni ou bilateral, não sendo verificada nenhuma outra alteração que contraindicasse a facectomia. Adicionalmente à opacidade da lente inerente à catarata, apenas dois olhos apresentaram degeneração vítrea com número inferior de cinco pontos ecogênicos na Câmara vítrea. Os dois cães que foram submetidos à cirurgia bilateral obedeceram a um intervalo de 90 dias, sendo que ambos os bulbos foram mantidos afácicos.

Os cães foram distribuídos em três grupos experimentais, designados: grupo $(\mathrm{G})$ de cães afácicos (CA), constituído de 11 bulbos oculares; grupo de cães pseudofácicos, de cinco bulbos oculares com implante de duas lentes intraoculares de silicone dobrável (AMO PhacoFlex II, SI 40 NB, AMO, SP-Brasil), em piggyback (PP); grupo de cães pseudofácicos, de cinco bulbos oculares, com implante de lente veterinária acrílica hidrofílica dobrável de $41 \mathrm{D}$ (C DOG SE, Corneal do Brasil, RJ) (PL).

Por solicitação do Serviço de Oftalmologia, ao completarem cinco anos de pós-operatório, todos os animais foram submetidos a exame oftálmico completo, seguido de exame ultrassonográfico (GE, modelo LOGIC 3, Brasil), com transdutor linear multifrequencial de $10 \mathrm{MHz}$ pelas técnicas transpalpebral e corneal, sob ação de anestésico tópico (Anestalcon - Alcon - Brasil), os quais foram realizados pelo mesmo profissional.

Avaliaram-se possíveis alterações nas estruturas internas do bulbo ocular, particularmente cápsula posterior da lente, lentes intraoculares implantadas com especial atenção ao seu posicionamento, câmara vítrea e retina.
Clinicamente, também foi avaliada a presença ou não de alterações como: hiperemia conjuntival, quemose, hifema, hipópio, uveíte, opacidade de cápsula posterior, posição da LIO, degeneração do humor vítreo (sinerese e hialose asteroide) e descolamento de retina.

O aspecto ultrassonográfico da cápsula posterior, preservada à facectomia, ou seja, quanto à ecogenicidade, que corresponde à opacidade capsular, obedeceu à classificação em escores de: 0 - ausente (com ausência de ecogenicidade); 1moderado (com presença de ecogenicidade); 2 severo (com presença de ecogenicidade avançada).

Quanto à posição da lente intraocular, foi classificada em: sem LIO (escore-0), posicionada (escore-1), descentrada (escore-2) ou deslocada para as câmaras anterior ou vítrea (escore-3).

Para a avaliação da câmara vítrea, foi aplicada a classificação descrita por Labruyère et al. (2008), a seguir: grau 0 - câmara vítrea completamente anecoica; grau 1 - degeneração vítrea média (menos de 10 pontos ecogênicos vistos na câmara vítrea); grau 2 - degeneração vítrea moderada (mais de 10 pontos ecogênicos, mas envolvendo subjetivamente menos que a metade da câmara vítrea com o bulbo ocular imóvel); grau 3 - degeneração vítrea acentuada (pontos ecogênicos envolvendo mais que a metade da câmara vítrea com o bulbo ocular imóvel).

O descolamento de retina também foi avaliado, sendo designado escore 0 para a ausência dele, escore 1 para descolamento total, e escore 2 para deslocamento parcial ou incompleto.

As mensurações das variáveis biométricas: comprimento axial, distância entre corpo ciliar na imagem ultrassonográfica em plano dorsal, câmara anterior e câmara vítrea foram realizadas pela imagem ultrassonográfica em plano dorsal do bulbo ocular (Fig. 1).

Para a comparação entre os grupos, utilizou-se a técnica de análise da variância para o modelo com um fator complementado com o teste de comparações múltiplas de Tukey (Zar, 2009), considerando-se o nível de significância de 5\%. 

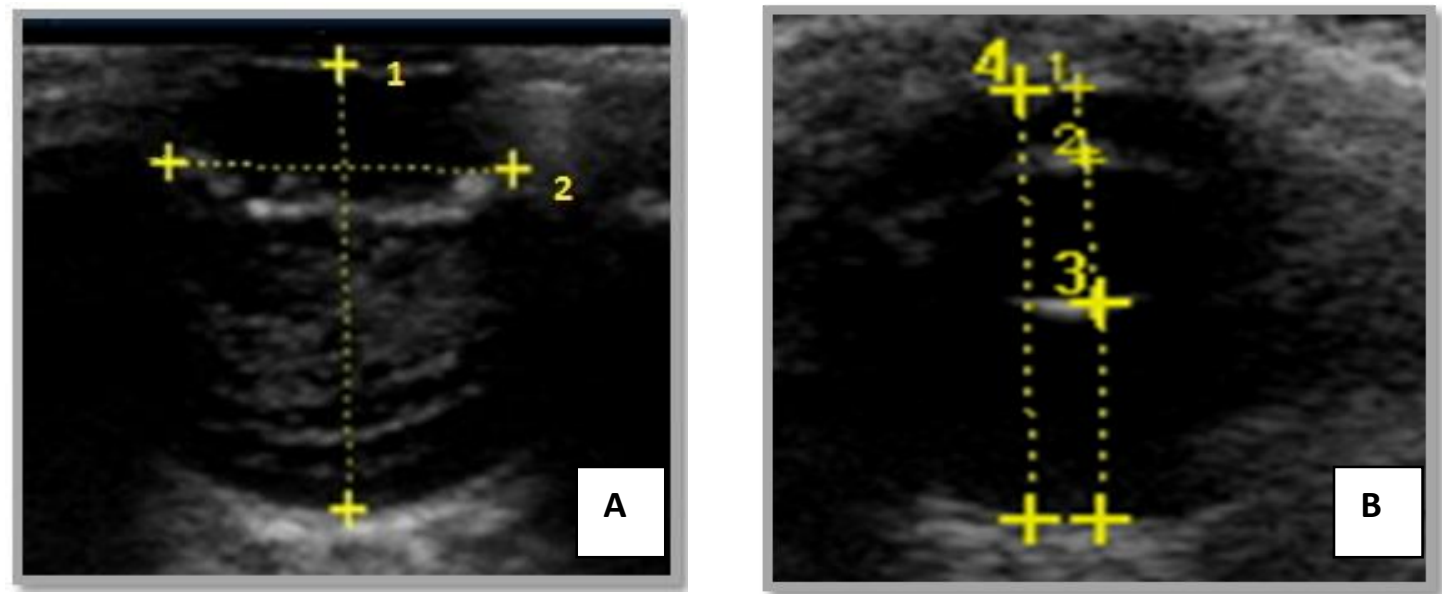

Figura 1. Mensurações das variáveis biométricas: Imagens ultrassonográficas em plano dorsal do bulbo ocular de cão do grupo CA. A. Observar a mensuração do comprimento axial em 1 e a distância, na imagem, entre o corpo ciliar em 2. B. mensurações: 1 - câmara anterior; 2 - lente; 3 - câmara vítrea; 4 comprimento axial.

\section{RESULTADOS E DISCUSSÃO}

As avaliações clínicas qualitativas do comportamento do bulbo ocular, que se relacionam diretamente ao tempo de pós-operatório, tais como secreção ocular, blefarospasmo, hiperemia conjuntival ou quemose, não foram observadas em nenhum dos animais examinados, por se tratar de ausência de quadro inflamatório e avaliação tardia.

Os exames ultrassonográficos foram facilmente exequíveis apenas com anestesia tópica em todos

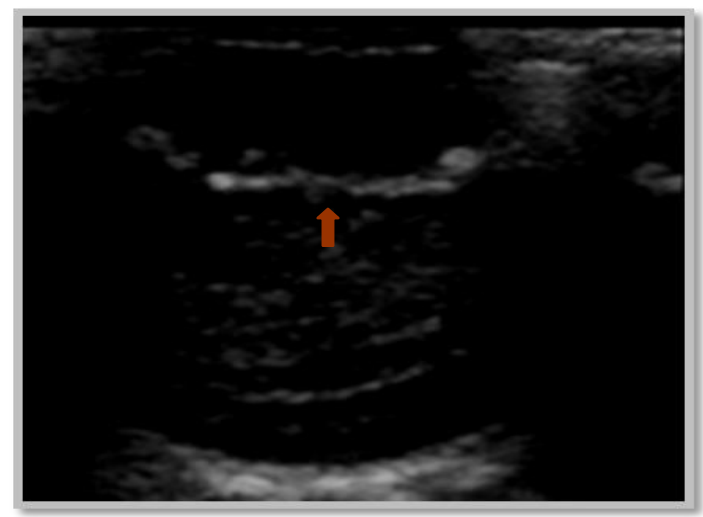

Figura 2. Imagem ultrassonográfica em plano dorsal do bulbo ocular de cão do grupo CA, onde se pode observar linha ecogênica irregular em região da lente, que representa cápsula posterior da lente (seta). os animais, de acordo com o descrito por vários autores (Gonzalez et al., 2001; Carvalho, 2004).

Pelas imagens obtidas ao exame ultrassonográfico, foi possível descrever a presença ou não da lente nos diferentes grupos estudados: grupo CA (Fig. 2), grupo PL (Fig. 3) e grupo PP (Fig. 4). A principal diferença na imagem ultrassonográfica entre os grupos com LIO ou pseudofácicos está na presença de reverberação (Fig.5) semelhante a um artefato dentro do vítreo, principalmente na LIO em piggyback.

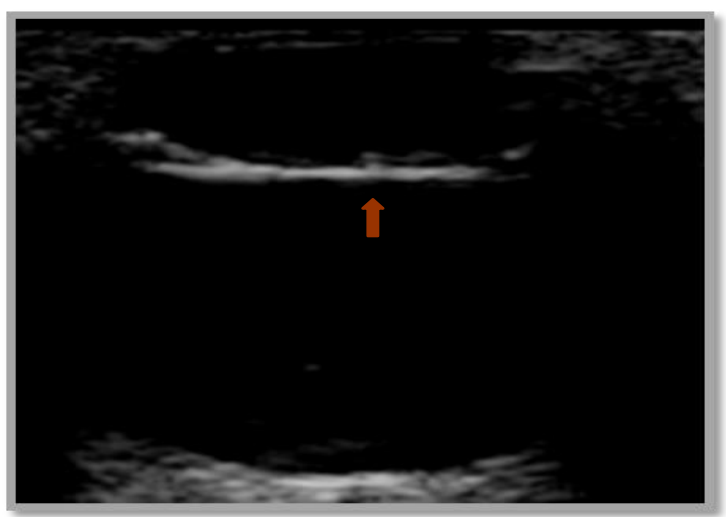

Figura 3. Imagem ultrassonográfica em plano dorsal do bulbo ocular de cão do grupo PL, onde se pode observar linha ecogênica retilínea em região da lente, que representa a LIO veterinária 41 D (seta). 


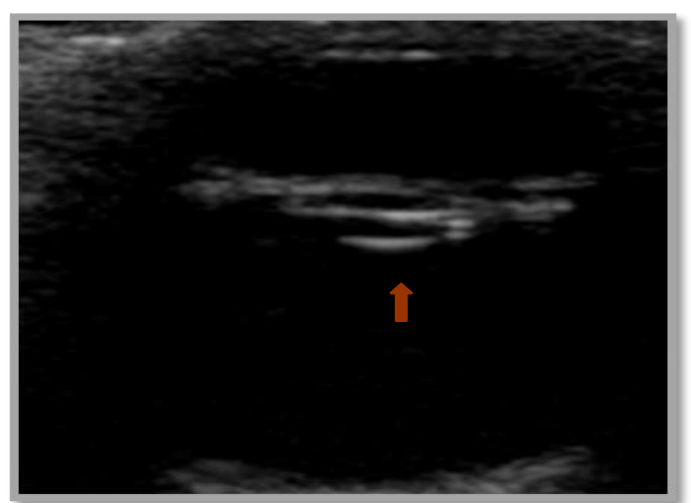

Figura 4. Imagem ultrassonográfica em plano dorsal do bulbo ocular de cão do grupo PP, onde se podem observar duas linhas ecogênicas paralelas em região da lente, que representam a LIO em piggyback (seta).

Ao se analisarem os achados ultrassonográficos relacionados à opacidade de cápsula posterior, independentemente dos grupos experimentais, observou-se que 33,33\% (7/21) não exibiram opacidade capsular, 23,80\% (5/21) apresentaram opacidade capsular discreta, a mesma porcentagem demonstrou opacidade capsular moderada e $19,04 \%$ (4/21) denotaram opacidade capsular severa. Quanto à posição da lente intraocular, dos 10 bulbos oculares em que foram implantadas LIO, em dois (20\%), pertencentes ao GPP, elas estavam descentradas e deslocadas para a câmara vítrea.

Considerando-se o segmento posterior, ao exame ultrassonográfico, visibilizaram-se três bulbos oculares com hialose asteroide, dois do GCA e um do GPL, dois com presença de coágulo/hemorragia, sendo um do GCA e outro do GPP, além de um animal com edema de papila GCA. Adicionalmente, foi observado o deslocamento da LIO para a câmara vítrea, em dois bulbos oculares do GPP, resultando, em sua grande maioria, ou seja, $80 \%$, implantes de LIO centralizados no momento do exame, o que confere com Yi et al. (2006), em cujo estudo dois de 32 bulbos oculares avaliados apresentaram descentralização da LIO. Segundo Reyes et al. (2001), 68,90\% das lentes luxadas para câmara vítrea foram secundários às complicações da facoemulsificação, como o mau posicionamento da LIO ou a contração capsular.

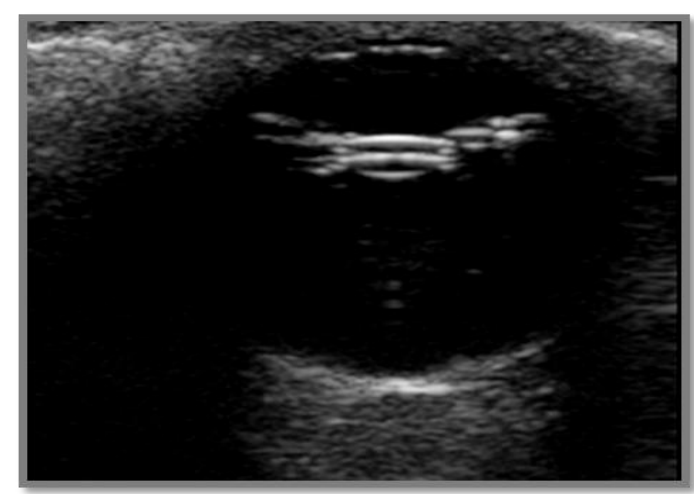

Figura 5. Imagem ultrassonográfica em plano dorsal do bulbo ocular de animal do grupo PP, da câmara vítrea classificada como degeneração vítrea média grau 1, onde se observam poucos pontos ecogênicos dispersos na câmara vítrea. Notar reverberação (seta), devido à presença da LIO em piggyback.

Quanto à avaliação da câmara vítrea, segundo a classificação de Labruyère et al. (2008), 33,33\% (7/21) foram classificadas como grau $0 ; 28,57 \%$ $(6 / 21)$ apresentaram grau $1 ; 19,04 \%$ (4/21) foram classificadas como grau 2; e a mesma porcentagem, ou seja, 19,04\% (4/21), foi classificada como grau 3.

Do total dos bulbos avaliados, sete não apresentaram degeneração vítrea e a maior concentração foi observada no grupo PL com $80 \%$ de bulbos oculares classificados como degeneração severa, ou seja, quatro de cinco avaliados, seguido do grupo PP com $60 \%$ de degeneração, porém o grupo que apresentou classificação mais severa foi o PL.

O descolamento de retina foi observado em três casos, sendo apenas um com descolamento total e dois com parcial; o grupo PL foi o único em que não foi observado nenhum dos casos.

A análise das variáveis biométricas, obtidas pela ultrassonografia ocular modo B, está apresentada na Tab. 1; verificou-se que, entre os grupos, não houve diferença significante $(p>0,05)$ quanto à média do comprimento axial e da câmara anterior; no entanto, a distância do corpo ciliar foi superior no GCA quando comparada aos pseudofácicos, tanto com implante duplo em piggyback (PP) quanto comparada com LIO de $41 \mathrm{D}$ (PL). A câmara vítrea também apresentou médias superiores no GPL, quando comparada ao GCA. 
Tabela 1. Média e desvio-padrão das variáveis biométricas nos diferentes grupos experimentais

\begin{tabular}{cccc}
\hline $\begin{array}{c}\text { Variável } \\
\text { Média } \pm \text { SD }\end{array}$ & CA & PL & PP \\
\hline $\begin{array}{c}\text { Comprimento } \\
\text { axial }\end{array}$ & $1,64 \pm 0,47 \mathrm{a}$ & $1,88 \pm 0,20 \mathrm{a}$ & $1,89 \pm 0,14 \mathrm{a}$ \\
\hline $\begin{array}{c}\text { Corpo } \\
\text { ciliar }\end{array}$ & $1,32 \pm 0,33 \mathrm{a}^{(1)}$ & $1,71 \pm 0,17 \mathrm{~b}$ & $1,61 \pm 0,15 \mathrm{~b}$ \\
\hline $\begin{array}{c}\text { Câmara } \\
\text { anterior }\end{array}$ & $0,44 \pm 0,15 \mathrm{a}$ & $0,48 \pm 0,24 \mathrm{a}$ & $0,54 \pm 0,15 \mathrm{a}$ \\
\hline $\begin{array}{c}\text { Câmara } \\
\text { vítrea }\end{array}$ & $0,83 \pm 0,36 \mathrm{a}$ & $1,01 \pm 0,12 \mathrm{ab}$ & $1,10 \pm, 23 \mathrm{~b}$
\end{tabular}

${ }^{(1)}$ Duas médias seguidas de pelo menos uma mesma letra igual não diferem entre si $(\mathrm{p}>0,05)$.

Considerando-se as alterações de hiperecogenicidade capsular, estas foram percentualmente maiores nos grupos com o uso da LIO, o que está de acordo com Yi et al. (2006), que encontraram em seu trabalho $34,37 \%$ de opacidade de cápsula posterior, mas difere de Kleiner (2007), o qual relatou que a grande maioria dos cães em que se implantou a LIO encontrou-se em perfeito estado visual, sem desconforto ocular e sem opacidades capsulares significativas; no entanto, sua avaliação não foi tardia, tratando-se de pós-operatório imediato, com no máximo 30 dias.

Quanto à avaliação da cápsula posterior da lente, conforme citado anteriormente, dos 21 bulbos oculares avaliados sete não apresentaram hiperecogenicidade capsular, entretanto destes, cinco eram do grupo CA $(45,45 \%)$; já dentro do grupo $\mathrm{PP}$, todos apresentaram hiperecogenicidade, que variou de leve a severa $(100 \%)$. No grupo PL, a maioria, três bulbos oculares $(60 \%)$, foi classificada com hiperecogenicidade capsular de moderada a severa, o que permite propor que a presença de duas LIOs pode acarretar em maior predisposição à opacidade capsular, que dificulta a acuidade visual no pós-operatório, conforme Rodrigues (2004), o qual observou maior opacidade com o uso de implante em piggyback aos 60 dias de pós-operatório em relação aos animais afácicos, o que resulta em hiperecogenicidade ao exame ultrassonográfico.

Em relação às medidas comprimento axial, profundidade de câmara anterior e câmara vítrea obtidas pela ultrassonografia ocular em modo B, os valores encontrados no presente estudo, independentemente do grupo, estavam de acordo com Williams e Wilkie (1996), que realizaram essas mensurações sem o uso de LIO.
No presente estudo, foram encontradas alterações em câmara vítrea compatíveis com degeneração, opacidades, exsudato ou hemorragia em 14 dos bulbos oculares examinados $(66,66 \%)$. Tais achados suportam os anteriormente encontrados por Woerdt et al. (1993), em que $23 \%$ dos pacientes com catarata estudados apresentavam alterações do vítreo. Woerdt et al. (1993) mostraram que o percentual de olhos com alterações no vítreo aumentava na razão direta da maturação da catarata.

Consoante o que descreveu Rodrigues Jr. (2008) quanto à correlação entre descolamento de retina e degeneração vítrea, ainda que alguns autores reconheçam como comuns os descolamentos de retina secundários à degeneração vítrea (Dziezyc, 1990; Woerdt et al., 1993; Narfström e Ekesten, 1998), não foram constatadas tais intercorrências diretas no presente estudo, pois dos três bulbos oculares com descolamento de retina, em apenas um foi notada degeneração vítrea classificada como leve, e nos outros ela era ausente.

No presente estudo, os resultados referentes às condições da câmara posterior dos bulbos oculares avaliados, principalmente aqueles com opacidades dos meios transparentes, em geral, não divergiram dos reportados na literatura que propõem a ultrassonografia como procedimento valioso e de grande significado na avaliação do bulbo ocular (Woerdt et al., 1993; Davidson e Nelms, 1998); no entanto, a maioria dos trabalhos relata achados pré-operatórios (Martins et al., 2010), havendo ainda escassez de avaliação ultrassonográfica pós-operatória, especialmente as que referem observações tardias dos procedimentos de facoemulsificação e implante de lentes intraoculares em cães; vale salientar que o implante da LIO, na medicina veterinária, 
apresenta-se como técnica com potencial crescimento e desenvolvimento, especialmente nos países desenvolvidos, mas também já iniciando no Brasil, sendo de fundamental importância a descrição de métodos de diagnóstico e alterações associadas.

\section{CONCLUSÕES}

O exame ultrassonográfico em modo B propicia acesso conveniente e não invasivo na avaliação do bulbo ocular e de LIOs no pós-operatório tardio da facoemulsificação, tornando possível descrever o implante e diferenciar as LIOs de aplicação única daquelas em piggyback, detectar luxações da lente, degeneração de vítreo e descolamento de retina. Tanto pela avaliação clínica quanto pela ultrassonográfica dos cães, aqueles com implante da LIO resultaram em maior número de alterações detectadas no pósoperatório tardio.

\section{REFERÊNCIAS}

BISTNER, S.I. Recent developments in comparative ophthalmology. Comp. Contin. Educ. Pract. Vet., v.14, p.1304-1323, 1992.

CARVALHO, C.F. Ultrassonografia em pequenos animais. São Paulo: ROCA, 2004. $365 \mathrm{p}$.

DAVIDSON, M.G.; NASISSE, M.P.; JAMIESON, V.E. et al. Phacoemulsification and intraocular lens implantation: a study of surgical results in 182 dogs. Prog. Vet. Comp. Ophthalmol., v.1, p.233-238, 1991.

DAVIDSON, M.G.; NELMS, S.R. Diseases of the lens and cataract formation. In: GELLAT, K.N. Veterinary Ophthalmology. 3.ed. Philadelphia: Lippincott Williams And Wilkins, 1998. p.818-828.

DIETRICH, U.M. Ophthalmic Examination and Diagnostics. In: GELATT, K.N. Veterinary Ophthalmology. 4.ed. Philadelphia: Blackwell Publishing, 2007. p.818-828.

DZIEZYC, J. Cataract surgery. Current approaches. Vet. Clin. North Am. Small Anim. Pract., v.20, p.737-54, 1990.

GELATT, K.N. Veterinary Ophthalmology. 4.ed. Philadelphia: Blackwell Publishing, 2007. 1669 p.
GLOVER, T.D.; CONSTANTINESCU, G.M. Surgery for cataract. Vet. Clin. North Am. Small Anim. Pract., v.27, p.1143-1173, 1997.

GONZALEZ, E.M.; RODRIGUEZ, A.; GARCIA, I. Review of ocular ultrasonography. Vet. Radiol. Ultrasound, v.42, p.485-495, 2001.

KEALY, J.K.; MCALLISTER, H.; GRAHAM, J.P. Radiografia e ultrassonografia do cão e do gato. 5.ed. Rio de Janeiro: Elselvier, 2012. 580p.

KLEINER, J.A. Implante de lente intraocular acrílica dobrável de 41D em cães após facoemulsificação. Acta Scient. Vet., v.35, p.623625, 2007.

LABRUYÈRE, J.J.; HARTLEY, C.; ROGERS, $\mathrm{K}$. et al. Ultrasonographic evaluation of vitreous degeneration in normal dogs. Vet. Radiol. Ultrasound, v.49, p.165-171, 2008.

LAUS, J.L.; CANOLA, J.C.; MAMEDE, F.V. et al. Orbital cellulites associated with Toxocara canis in a dog. Vet. Ophthalmol., v.6, p.333-336, 2003.

MARTINS, B.C.; LIMA, F.S.; LAUS, J.L. Simultaneous mode A and mode B echobiometry of senile cataractous eyes in dogs. Arq. Bras. Med. Vet. Zootec., v.62, p.42-46, 2010.

NARFSTRÖM, K.; EKESTEN, B. Diseases of canine ocular fundus. In: GELLAT, K. N. Veterinary Ophthalmology. 3.ed. Philadelphia: Lippincott Williams and Wilkins, 1998. p.860873.

NASISSE, M.P.; DAVIDSON, M.G.; JAMIESON, V.E. et al. Phacoemulsification and intraocular lens implantation: a study of technique in 182 dogs. Prog. Vet. Comp. Ophthalmol., v.1, p.225232, 1991 .

NYLAND, T.G.; MATTOON, J.S. Ultrassom diagnóstico em pequenos animais. 2.ed. São Paulo: Roca, 2005. 469p.

PAIVA, S.C.C.S. Caracterização das manifestações oftálmicas odontogênicas em cães e gato. 2011. 126f. Dissertação (Mestrado em Medicina Veterinária) - Departamento de Medicina Veterinária, Universidade Federal do Paraná, Curitiba. 
PEIXOTO, T.P.; RANZANI, J.J.T.; BRANDÃO, C.V.S. et al. Análise da fórmula SRK/T no cálculo de lente intra-ocular em cães portadores de catarata. Arq. Bras. Med. Vet. Zootec., v.60, p.1418-1425, 2008.

PONTES, L.L.; CORRÊA, F.G. Métodos de diagnóstico por imagem em cães com catarata. Rev. Cient. Eletron. Vet., v.9, p.16, 2011.

REYES, M.A.Q.; SANTIAGO, N.J.S.; WIECHERS, F.G. et al. Abordaje quirúrgico del lente luxado a cavidad vítrea. Rev. Mex. Oftalmol., v.75, p.91-97, 2001.

RODRIGUES, G.N. Estudo clínico da facoemulsificação em cães, com e sem implante de lente intraocular em piggback. 2004. 175f. Tese (Doutorado em Medicina Veterinária) Faculdade de Medicina Veterinária e Zootecnia, Universidade Estadual Paulista, Botucatu.

RODRIGUES JUNIOR, E.F. Ultrassonografia pré-cirúrgica da lente e do segmento posterior de cães portadores de catarata. 2008. 68f. Dissertação (Mestrado em Medicina Veterinária) - Faculdade de Ciências Agrárias e Veterinárias, Universidade Estadual Paulista, Jaboticabal.

SLATTER, D. Fundamentos de Oftalmologia Veterinária. 3.ed. São Paulo: Roca, 2005. 686p.
SQUARZONI, R.; MORALES, M.S.A.; SAFATLE, A.M.V. et al. Avaliação ultra-sonográfica do segmento posterior de olhos de cães diabéticos e não diabéticos portadores de catarata. Pesqui. Vet. Bras., v.27, p.455-461, 2007.

WHITLEY, R.D.; MCLAUGHLIN, S.A.; WHITLEY, E.M. et al. Cataract removal in dogs: The surgical techniques. Vet. Med., v.88, p.859-866, 1993.

WILLIAMS, J.; WILKE, D.A. Ultrasonography of the eye.A ultrassonografia do bulbo ocular. Comp Contin Ed Pract Vet 1996;18:826-830. Comp. Contin. Educ. Pract. Vet. Educ., v.18, p.826-830, 1996.

WILLIAMS, D.L.; BOYDELL, I.P.; LONG, R.D. Current concepts in the management of canine cataract: a survey of techniques used by surgeons in Britain, Europe and the USA and a review of recent literature. Vet. Rec., v.138, p.347-353, 1996.

WOERDT, A.V.D.; WILKIE, D.A.; MYER, W. Ultrasonographic abnormalities in eyes of dogs with cataracts: 147 cases (1986-1992). J. Am. Vet. Med. Assoc., v.203, p.838-841, 1993.

YI, N.Y.; PARK, S.A.; JEONG, M.B. et al. Phacoemulsification and acryl foldable intraocular lens implantation in dogs: 32 dogs. $J$. Vet. Sci., v.7, p.281-285, 2006.

ZAR, J.H. Biostatistical analysis. 4.ed. New Jersey: Prentice Hall, 1999. 